\section{Evaluation of apoptotic cell death in normal and chondrodystrophic canine intervertebral discs}

\author{
Marie Klauser, ${ }^{1}$ Franck Forterre, ${ }^{1}$ \\ MarCus Doherr,2 Andreas Zurbriggen, ${ }^{2}$ \\ David Spreng, 1 Simone Forterre ${ }^{1}$ \\ 1Division of Small Animal Surgery and \\ Orthopedics, Department of Clinical \\ Veterinary Medicine; ${ }^{2}$ Division of Clinical \\ Research, Department of Clinical \\ Research and Veterinary Public Health, \\ Vetsuisse Faculty Bern, University of \\ Bern, Switzerland
}

\section{Abstract}

Disc degeneration occurs commonly in dogs. A variety of factors is thought to contribute an inappropriate disc matrix that isolate cells in the disc and lead to apoptosis. Disc herniation with radiculopathy and discogenic pain are the results of the degenerative process. The objective of this prospective study was to determine the extent of apoptosis in intact and herniated intervertebral discs of chondrodystrophic dogs and non-chondrodystrophic dogs. In addition, the nucleus pulposus (NP) was histologically compared between non-chondrodystrophic and chondrodystrophic dogs. Thoracolumbar intervertebral discs and parts of the extruded nucleus pulposus were harvested from 45 dogs. Samples were subsequently stained with haematoxylin-eosin and processed to detect cleaved caspase-3 and poly(ADP-ribose) polymerase. A significant greater degree of apoptosis was observed in herniated NPs of chondrodystrophic dogs compared to non- chondrodystrophic dogs with poly (ADP-ribose) polymerase and cleaved caspase3 detection. Within the group of chondrodystrophic dogs, dogs with an intact disc and younger than 6 years showed a significant lower incidence of apoptosis in the NP compared to the herniated NP of chondrodystrophic dogs. The extent of apoptosis in the annulus fibrosus was not different between the intact disc from chondrodystrophic and non- chondrodystrophic dogs. An age-related increase of apoptotic cells in NP and annulus fibrosus was found in the intact non-herniated intervertebral discs. Histologically, absence of notochordal cells and occurrence of chondroid metaplasia were observed in the nucleus pulposus of chondrodystrophic dogs. As a result, we found that apoptosis plays a role in disc degeneration in chondrodystrophic dogs.

\section{Introduction}

Disc herniation is the most common spinal neurological problem in dogs, ${ }^{1}$ occurring occasionally as a consequence of traumatic injury but most frequently as a sequela to disc degeneration. Degeneration of the intervertebral disc (IVD) is observed with aging 2,3 and in chondrodystrophic breeds during growth.1,4 The degeneration process begins in the nucleus pulposus, where it is associated with a loss of water due, in part, to the quantitative diminution in levels of the proteoglycans (aggrecan) which provide hydration properties to the IVD and an increase in collagen $1,2,4,5$ resulting in impaired nutritional intake. 6

In chondrodystrophic (CD) dogs, this catabolic process begins at three months of age. In the newborn, the disc surface in CD dogs is similar to that of non-chondrodystrophic (nonCD) dogs but it contains an abnormal perinuclear zone consisting of immature fibrocartilage. A degeneration occurs rapidly and simultaneously in all discs along the entire vertebral column, leading to morphologic changes within the nucleus pulposus (NP), which calcifies following an abnormal proliferation of chondrocytes. ${ }^{7,8}$ Furthermore, loss of notochordal cells, which synthesize growth factors for the maintenance of the disc, occurs much earlier in CD than in other breeds. ${ }^{9}$ In consequence, the IVD is less protected against the development of degenerative processes. ${ }^{10,11}$

In humans, apoptosis is considered to play an important role in the degeneration of IVDs. ${ }^{3,12-16}$ To the authors' knowledge, however, apoptosis has not been investigated in degenerated or herniated discs in dogs. This mechanism of cell death, characterized by DNA fragmentation, chromatin condensation, membrane blebbing, formation of apoptotic bodies and phagocytosis by macrophages or neighboring cells, takes place as a physiological occurrence in the regulation of cell turnover and in pathological processes. ${ }^{17,18}$ Central to the process of apoptosis is the activation of specific proteases, particularly caspases, by intrinsic pathways, such as following DNA damage, as well as by extrinsic pathways, such as through TNF- or FAS-ligand receptor binding. ${ }^{14-16,18}$ Caspases are present in essentially all viable cells as inactive pro-enzymes. Activation by cleavage of some caspases, known as initiator caspases, is an early event in apoptosis. Following activation of caspases cell death cannot be reversed..$^{19}$ Caspase- 3 is a protease that degrades a variety of proteins involved in cell maintenance and repair and is recognized as a central player in mediating apoptosis. Detection of cleaved caspase-3 in tissues using a specific antibody can therefore be used to demonstrate apoptosis. ${ }^{20}$
Correspondence: Franck Forterre, Division of Small Animal Surgery and Orthopedics, Department of Clinical Veterinary Medicine, University of Bern, Länggassstrasse 128, 3012 Berne, Switzerland.

Tel. +41.31.631.2401 - Fax: +41.31.631.2275.

E-mail: franck.forterre@vetsuisse.unibe.ch

Keywords: apoptosis, disc herniation, chondrodystrophic, dog

Contributions: MK, analysis and interpretation of data, drafting the article; FF, design, interpretation of data, revising article; MD, analysis of data, revising article; AZ, support of experiments, revising article; DS, design and revising article; SF, analysis and interpretation of data, drafting article, tables and figures.

Conflict of interest: none of the authors has any financial or personal relationships that could inappropriately influence or bias the content of the paper.

Received for publication: 12 October 2011. Revision received: 22 December 2011.

Accepted for publication: 23 December 2011.

This work is licensed under a Creative Commons Attribution NonCommercial 3.0 License (CC BYNC 3.0).

(C) Copyright M. Klauser et al., 2012 Licensee PAGEPress srl, Italy

Veterinary Science Development 2012; 2:e6 doi:10.4081/vsd.2012.e6

Furthermore, caspase activation results in the cleavage of the DNA repair enzyme, poly (ADP-ribose) polymerase (PARP) into two characteristic fragments of 85 and $25 \mathrm{kDa}$. Detection of the $85 \mathrm{kDa}$ PARP fragment by specific antibody can therefore also be used to demonstrate apoptosis in tissues. The main goal of the present study was to investigate apoptosis by cleaved caspase- 3 and PARP detection in canine intervertebral discs. The study comported three related parts.

The first objective was to determine the extent of apoptosis in the NP in herniated discs of chondrodystrophic dogs (herniated CD dogs) and to compare the apoptotic activity with that of two age groups of non-herniated discs in chondrodystrophic dogs (non-herniated CDyoung, non-herniated CDold) and a group of intact discs in non-chondrodystrophic dogs (non-CD dogs). A second objective was to compare the extent of apoptosis in the annulus fibrosus (AF) in non-herniated CD and non-CD dogs. A third objective was to compare the microscopic appearance of the NP among the groups. 


\section{Materials and Methods}

\section{Animals and sample collection}

Dogs were recruited from client-owned animals presented to our referral institution. Herniated IVD material was harvested from CD dogs that were presented with an acute episode of Hansen type I thoracolumbar disc herniation (herniated CD group).The amount of AF harvested during the pilot study was not sufficient for apoptosis measurement and therefore only NP was examined in the herniated CD group. The extruded nucleus pulposus material was removed during surgery and immediately fixed in $10 \%$ neutral buffered formalin. Dogs were included in this group if they met the inclusion criteria of a complete clinical neurological and an MRI examination leading to a diagnosis of Hansen type I herniation between T12 and L 2 confirmed by thoracolumbar disc surgery. Intact non-herniated IVDs were harvested from non-CD and CD dogs immediately after euthanasia. Attached vertebral body segments of the IVDs at both ends were trimmed and discarded. These IVDs consist of the annulus fibrosus (AF) and the nucleus pulposus (NP) from the T12-T13, T13L1 and L1-L2 disc. These discs were chosen in accordance to the location of disc extrusion in the herniated CD group. In all discs except in the herniated CD group, in which this was not possible, the orientation of tissue sectioning was sagittal. Disc material was fixed in $10 \%$ neutral buffered formalin immediately after harvesting. All specimens were embedded in paraffin, sagittaly sectioned and processed for immunohistochemistry. The non-herniated CD dog group consisted of two age-matched subgroups: dogs 6 years or younger (non-herniated CDyoung) and dogs 8 years or older (nonherniated CDold). The control group consisted of various non-chondrodystrophic breeds (nonCD). Dogs of the three last groups had no previous history of back-related disorders and were euthanized for other reasons.

\section{Apoptosis detection by cleaved caspase-3 immunochemistry}

After removal of the paraffin-embedding medium, the sections were rehydrated in graded alcohol. Epitope retrieval was performed in heated $10 \mathrm{mM}$ sodium citrate buffer $(\mathrm{pH} \mathrm{6.0)}$ for $10 \mathrm{~min}$. Endogenous peroxidase activity was quenched by incubation of the chilled slides with a peroxidase-blocking solution ( $3 \%$ $\mathrm{H} 202$ in methanol). The slides were then blocked with $5 \%$ goat serum in phosphate buffered saline (PBS). According to a previous study, ${ }^{21}$ the specimens were treated with an anti-rabbit polyclonal antibody for caspase-3b diluted in PBS (1:125) and incubated overnight at $4^{\circ} \mathrm{C}$. After incubation with the first antibody, the slides were treated in the same way as slides after the PARP antibody described below.

\section{Apoptosis detection by PARP immunochemistry}

The paraffin-embedding medium was removed in xylene and the sections were rehydrated in graded alcohols. The slides were placed first in distilled water and then in PBS. Sections were permeabilized by incubation in $0.2 \%$ Triton X-100 in PBS followed by rinsing three times with PBS. Endogenous peroxidase activity was quenched by incubation with a peroxidase-blocking solution $\left(3 \% \mathrm{H}_{2} \mathrm{O}_{2}\right.$ in methanol). The sections were blocked with $5 \%$ goat serum in PBS. After removing the blocking solution, sections were incubated with the primary antibody overnight at $4^{\circ} \mathrm{C}$. According to the study of Krayer at al. (2008), the antibody used was a human polyclonal anti-PARP p85 fragment antibody diluted 1:100 in PBS. After rinsing in PBS, the slides were treated with a commercially available detection kit and closely monitored under light microscopy until a distinct positive signal appeared on the positive control tissue. After a last washing step in distilled water, the slides were counterstained with hematoxylin and mounted for evaluation. In parallel, negative control slides were handled and prepared in the same way as the other slides by omitting the primary antibody or replacing with the negative control rabbit immunoglobin fraction diluted to the same protein concentration as the primary antibody. Sections of healthy canine lymph nodules were used as positive controls. Several slides were made from each tissue sample, such that 3 slides were stained with each antibody for all samples harvested.

\section{Apoptotic cell counts}

The stained tissue slides were evaluated under light microscopy by the same observer (M.K.). Ten randomly selected view fields were scored for each slide at $200 \mathrm{x}$ magnification and the percentage of cells stained positive for caspase-3 or PARP out of total cells were calculated. Apoptosis in the AF and NP was classified into one of five categories: 0 ( $<5 \%$ apoptotic cells), 1 (5-10\% apoptotic cells), 2 (10$25 \%$ apoptotic cells), 3 (25-50\% apoptotic cells) or 4 (50-100\% apoptotic cells). For each disc sample-antibody combination, three slides were scored and apoptotic classification was based on the median and range of the three samples.

\section{Histology}

Routine histological sections were performed on disc specimens to evaluate the cellular content of the tissue. After embedding,
$4 \mu \mathrm{m}$ sections were cut, mounted on slides and stained with Mayer's haematoxylin-eosin. The quantity of chondrocyte-like cells, fibrocytes and notochord cells were subjectively evaluated and reported.

\section{Statistical analyses}

Statistical methods used were calculation of Spearman correlation and, as they were not normally distributed, the non-parametric Kruskal Wallis ANOVA on Ranks and, where appropriate, the Bonferroni correction for multiple comparison. A P value of $<0.05$ was considered statistically significant.

\section{Results}

\section{Grouping of dogs}

The herniated CD group consisted of 14 dogs (9 Dachshunds, 1 Papillon, 1 Coton de tulear, 1 French bulldog, 1 Japanese chin and 1 American Cocker spaniel). The Papillon and Coton de tulear were grouped to the CD dogs due to the degeneration characteristics of their IVD although they are not mentioned explicitly as chondrodystrophic breeds in the literature. Sex distribution was 7 males and 7 females and the median age 7 years (range 213). The non-herniated CD dogs were dived up into two subgroups due to the bimodal distribution of their apoptotic activity in the NP and AF as followed: 8 non-herniated CDold dogs: 7 beagles and 1 miniature poodle, 5 males and 3 females, median age 9 (range 8-13 years); and 8 non-herniated CDyoung dogs: 8 beagles, 2 males and 6 females, median age 5 (range $3-6$ years). The non-CD dogs consisted of 15 dogs of various non-chondrodystrophic breeds (3 Leonbergers, 2 Huskies, 2 Labrador retrievers, 2 Border collies, 3 Greyhounds, 3 mongrel dogs), 8 males and 7 females, median age 6 (range 1-11 years). Considering all groups, no statistical difference in apoptotic activity between males and females was found (data not shown).

\section{Apoptotic activity}

A comparison of the apoptotic activities showed a strong statistical correlation between both antibodies used ( $r s=0.87$ ). A statistical significant age-related dependency in apoptotic activity in disc tissue was found for the $\mathrm{CD}$ dog group ( $\mathrm{rs}=0.80$ for NP over all subgroups, rs $=0.71$ for AF in the non-herniated CD dog group). Caspase- 3 activity and PARP positivity were significantly higher in the NP of herniated CD dogs and non-herniated CDold dogs compared to the groups of non-herniated CDyoung and non-CD dogs $(\mathrm{P}=0.002)$ (Figure 1). In contrast, the apoptotic activity was not statistical different between the two groups, 
herniated CD and the non-herniated CD old (Table 1). No difference in apoptotic activity was found in the AF between the non-herniated CDold and the non-CD dogs. In contrast, regarding the $\mathrm{CD}$ subgroups there was a significant higher apoptotic activity in the AF of old (non-herniated CDold) compared to the young dogs grouped as non-herniated CD young. As AF was not sampled in the herniated CD group, apoptotic activity could not be evaluated in the AF in this group.

\section{Histology}

In chondroid metaplasia, a proliferation of chondrocyte-like cells was observed particularly within the NP of CD dogs (Figure 1C, E, F), leading to secondary calcification. All samples from CD dogs older than 1 year of age were devoid of notochordal cells. In contrast, notochordal cells were detected in the histological preparations of the NP in non-CD dogs up to an age of 9 years (Figure 2). Groups of chondrocyte-like cells were observed in all NP samples of CD dogs (Figure 3). The older the dogs of the non-herniated CD group, the more chondrocyte-like cells were stained positive for apoptosis (Figure 1E, F).

\section{Discussion}

Findings of this study demonstrate the presence of apoptotic cell death in IVDs of both CD and non-CD dogs. Significantly higher levels of apoptosis were detected in non-herniated CD old dogs and CD dogs with IVD herniation. To our knowledge, no previous study has investigated cell viability in canine IVDs. Previous studies of the NP in CD dogs have demonstrated alterations in matrix constituents, such as increased proportions of collagen, 4 and decreased amounts of glycosaminoglycans ${ }^{22}$ and proteoglycans. ${ }^{5}$ Some authors also observed modifications in the cellular content within the NP of CD dogs, in particular, a decreased number of notochord cells ${ }^{10,11}$ and chondroid metaplasia.7,23 In one study, cell death was described in the NP but no discrimination between apoptosis and necrosis was made. ${ }^{7}$ In the present study, we demonstrated apoptosis in canine IVDs based on two specific immunochemical techniques (cleaved caspase-3 and PARP). Using both antibodies reinforces the reliability of results. ${ }^{21,24} \mathrm{~A}$ further method, frequently employed in apoptosis studies, is labeling of DNA strand breaks with TUNEL. ${ }^{12,18}$ However, this method is doubtable to allow differentiation between necrosis and apoptosis. $^{24}$ In fact of this, TUNEL was not adopted in the present study. Likewise, in recent human literature components of the extrinsic apoptotic pathway (TRAIL, Receptor DR4) were evaluated. 25,26 On the contrary, the
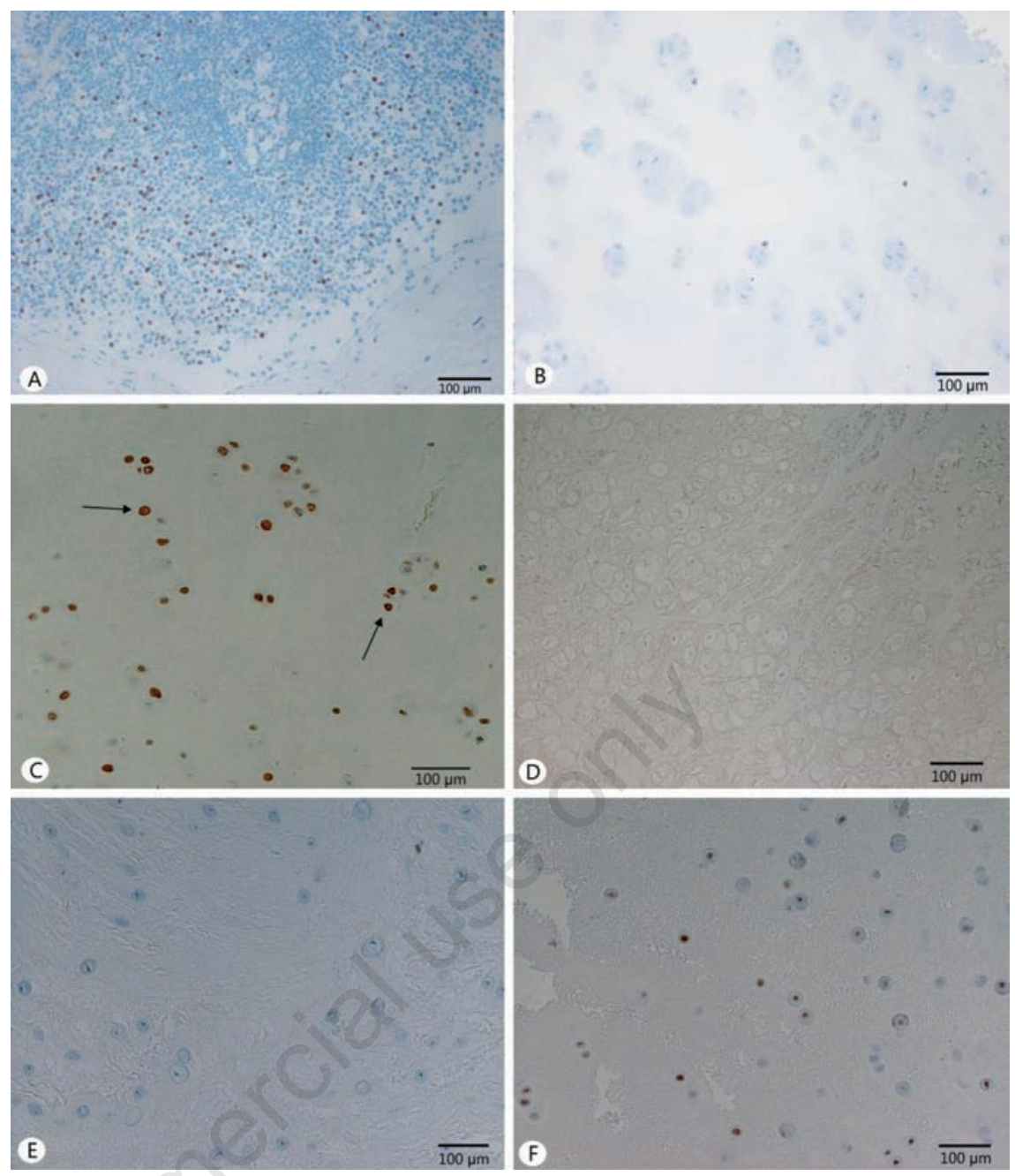

Figure 1. Photomicrographs of representative tissue samples immunostained by cleaved caspase-3 (A-D) and poly(ADP-ribose) polymerase (E, F). (A) Lymphnode stained with cleaved caspase-3 as positive control. (B) Negative control of a nucleus pulposus in a CD dog. (C) Herniated nucleus pulposus in a CD dog (Dachshound, 9 years). Caspase-3 positive cells show a brown staining. Chondrocyte-like cells (arrows) stained mostly positive indicating apoptosis. (D) No positive staining for cleaved caspase-3 of a nucleus pulposus in a non-CD dog (Labrador, 6 years); no chondrocyte-like cells are observable. Nucleus pulposus of non-herniated CD discs stained against Poly (ADP-ribose) polymerase in a 4 years old (E) and in a 10 years old Beagle (F). Chondrocyte-like cells are observable in $\mathrm{E}$ and $\mathrm{F}$, but only in (F) with a positive staining for apoptosis.

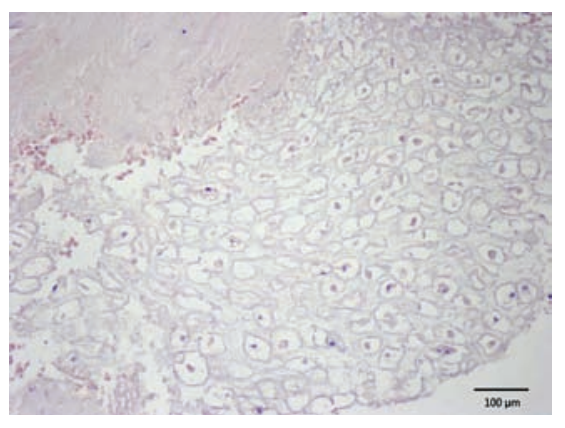

Figure 2. Photomicrograph of the nucleus pulposus in a non-chondrodystrophic dog (non-CD dog; 9 years old Greyhound) after Hematoxylin \& Eosin staining. Notochord cells are present in great numbers.

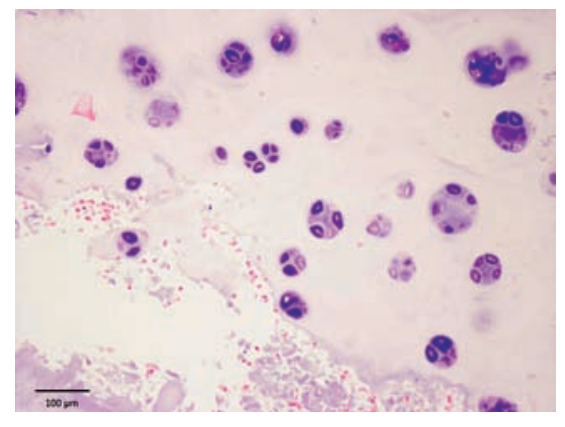

Figure 3. Photomicrograph of the nucleus pulposus in a non-herniated chondrodystrophic dog (non-herniated CDold dog; 9 years old Beagle) after H\&E staining: the nucleus pulposus contains exclusively chondroid metaplastic cells (chondrocytelike cells) grouped in clusters. 
detection of cleaved caspase- 3 which is activated at the end of both signaling cascades offers the advantage to include the intrinsic and extrinsic apoptotic pathway.

Since the induction of apoptosis is not synchronous throughout a tissue, cells at different stages of apoptosis coexist in tissue section. Generally, the time from initiation of apoptosis to completion can occur as quickly as 2-3 hours, ${ }^{27}$ however, in non-vascularized IVDs phagocytosis of apoptotic cells is delayed. During the apoptotic process, some proteins, such as caspases, are expressed only transiently. In consequence, the detection of apoptotic cells by caspase or PARP immunochemistry is only possible during a short window of time in which these proteins are present and not degraded due to the proceeded apoptotic process or the autolytic processes after euthanasia. In order to enumerate apoptotic cells in vivo in a realistic fashion, the duration of time between tissue harvesting and embedding (blocking cell metabolism and protein degradation) should be maintained as short as possible to avoid a false negative as well as a false positive assay during tissue preparation. ${ }^{18}$ For this reason, we arbitrarily decided to limit this duration to a maximum of 4 hours. However, further studies, focusing on the effect of time on apoptosis prior to paraffin embedding, are necessary to determine the optimal time span between tissue harvesting and embedding.

In the present study, apoptosis was present in the IVDs of both non-CD and CD dogs. A relationship between apoptosis and the pathophysiology of chondrodystrophism (chondroid metaplasia and calcification of intervertebral discs) has not been established. ${ }^{7}$ However, a strong relationship between the age of the CD dogs and the level of apoptosis was observed. This finding is in agreement with previous results regarding the degeneration of the disc with aging. $2,28,29$

One finding of our study was that the cells of the NP, which preserve the matrix, ${ }^{10}$ showed no apoptotic activity in IVDs of non-CD dogs. These cells, referred to as notochordal cells, arise from embryonic tissue and play an important role in the formation of the spine and the NP. ${ }^{30}$ However, the exact role that notochordal cells play in forming the nucleus pulposus is still largely unknown. Some authors have suggested that these cells directly synthesize the nucleus and differentiate into a chondrocytic cell type. ${ }^{31}$ Others have suggested that the nucleus is instead synthesized by cells that migrate from the surrounding mesenchyme, and that the notochordal cells direct cell migration and matrix synthesis and then undergo necrosis or apoptosis once disk formation is complete. $^{32,33}$ A loss of this cell population has been described during the first 60 months of life in non-CD dogs and before 12 months in

Table 1. Apoptotic activity in the nucleus pulposus and annulus fibrosus of the different groups detected by cleaved caspase- 3 and poly (ADP-ribose) polymerase (PARP) immunohistochemistry.

\begin{tabular}{|c|c|c|c|c|c|}
\hline Dogs & & $\begin{array}{c}\text { CD } \\
\text { herniated CD } \\
\text { dogs } \\
\mathrm{N}=14\end{array}$ & $\begin{array}{c}\text { non-herniated } \\
\mathrm{CD}_{\text {old dogs }} \\
\mathrm{N}=8\end{array}$ & $\begin{array}{c}\text { non-herniated } \\
\text { CD }_{\text {young dogs }} \\
\mathrm{N}=8\end{array}$ & non-CD \\
\hline Age & & $7(7 / 7)$ & $9(8 / 10)$ & $5(4 / 5)$ & $6(5 / 7)$ \\
\hline Nucleus pulposus & $\begin{array}{l}\text { Caspase } \\
\text { PARP }\end{array}$ & $\begin{array}{l}3^{*}(3 / 3) \\
3^{*}(3 / 4)\end{array}$ & $\begin{array}{l}2.75^{*}(2.5 / 3) \\
2.75^{*}(2.5 / 3)\end{array}$ & $\begin{array}{l}0^{\circ}(0 / 1) \\
0^{\circ}(0 / 1)\end{array}$ & $\begin{array}{l}1^{\circ}(0 / 1) \\
1^{\circ}(0 / 1)\end{array}$ \\
\hline Annulus fibrosus & $\begin{array}{l}\text { Caspase } \\
\text { PARP }\end{array}$ & $\begin{array}{l}\text { n.d. } \\
\text { n.d. }\end{array}$ & $\begin{array}{c}2.5^{*}(2 / 3) \\
3^{*}(3 / 3)\end{array}$ & $\begin{array}{l}0^{\circ}(0 / 1) \\
0^{\circ}(0 / 1)\end{array}$ & $\begin{array}{l}2^{*}(1 / 2) \\
2^{*}(1 / 2)\end{array}$ \\
\hline
\end{tabular}

Apoptosis was classified into one of five categories as described in the Materials and Methods section: 0 ( $<5 \%$ apoptotic cells), 1 (5-10\% apoptotic cells), 2 (10-25\% apoptotic cells), 3 (25-50\% apoptotic cells) or 4 (50-100\% apoptotic cells). Values are medians (lower/upper 95\% confidence intervals). Differences in superscripted symbols $\left({ }^{*}\right.$ and $\left.{ }^{\circ}\right)$ denote significant difference among the groups: $P \leq 0.01$. PARP, poly(ADP-ribose) polymerase; $\mathrm{CD}$, chondrodystrophic; n.d., not determined.

CD dogs. 9,34 Although no quantitative evaluation of these cells was undertaken in the present study, their presence in our dogs up to an age of 9 years may suggest that notochordal cell loss could be less than previously thought. Accordingly to the study from Erwin et al. 2008,35 we found that non-CD dogs maintained notochordal cells within their intervertebral disc with advancing age (Figure 2) and they are not known to develop degenerative disc disease until much later in life. In contrast, in our CD dogs we were not able to identify this cell population confirming the statement of Erwin et al. 2008 that they have a paucity of such notochord cells. Our CD dogs had a mean age of 7 years. Even the youngest dogs of the CD group were older than 12 months of age which is the reported onset of loss of notochordal cells (6 to 12 months of age). ${ }^{9,34}$ The driving force for the earlier loss of notocordal cells in CD dogs to date is unknown and it is presently unclear whether this disappearance is due to the terminal differentiation of the notochordal cells into cells with a chondrocytic phenotype, to programmed cell death, or to some other process. Further studies have to prove if a relationship exists between the lack of notochordal cells, chondrodystrophism and the development of disc disease which occurs much earlier in CD dogs.

Differences in the pathophysiology of degeneration between CD and non-CD dogs have been described. Results of previous studies suggest that the principal changes in the IVD in CD dogs occur within the nucleus pulposus. ${ }^{7}$ Indeed, Hansen ${ }^{34}$ made a histological distinction between chondrodysplastic and non-chondrodysplastic degeneration; the first being a rapid process of chondroid metaplasia, and the second a slow progressive process of fibroid metaplasia. In the light of our observations, a direct relationship between calcification and cellular apoptosis cannot be assumed and the role of mineralization in apoptosis remains unclear.

Degeneration of IVDs may lead to disc her- niation. Damage to or a break in the AF may lead to either disc extrusion (Hansen type I) or protrusion (Hansen type II) of the NP within the vertebral canal. In contrast, in CD dogs, primary signs of disc degeneration occur within the nucleus and a break in the AF is a secondary finding, ${ }^{7}$ which may lead to an extrusion (Hansen type I) of the NP. Our data presents no differences of the apoptotic extent in the $\mathrm{AF}$ of intact discs from $\mathrm{CD}$ and non-CD dogs. Only in the younger dogs fewer amounts of apoptosis was present. This supports that a normal level of apoptosis in the AF occurs as a physiological process to eliminate aged and injured cells and increases with age in the CD dogs. Findings in the present study of increased apoptosis in the NP in CD dogs are consistent with this mechanism of degeneration. In our study, samples of the AF were not collected in the herniated CD dog group. The authors plan a further study to examine apoptosis in this tissue and compare results with those of the present study.

The hypothesis that apoptosis could be enhanced by herniation ${ }^{18}$ was not corroborated by the results of the present study. Indeed, apoptotic activity was similar in IVD material from herniated and non-herniated CD dogs suggesting that apoptosis is present prior to disc herniation in dogs and is not influenced by the process of herniation itself and on the other hand do not correlate with the severity of discal degeneration. This corroborates the findings of a study on human IVDs, which failed to prove a linkage between level of apoptosis and degeneration. ${ }^{36}$ In studies of human IVDs, some authors correlated the degree of apoptosis with patient age. ${ }^{3,13,18,29}$ In our CD dog population, we could establish a similar correlation. However, the small number of dogs and the absence of age-matched subgroups in all groups do not allow extrapolating these results. Further studies with a greater number of age-matched patients are needed to evaluate the effect of age on apoptosis in canine IVDs. 
In conclusion, apoptosis plays a role in IVDs of CD dogs and especially in older dogs and in dogs with IVD hernia. These are preliminary findings and further studies, investigating apoptosis, degeneration and inflammation in the IVD are necessary to develop a more complete understanding of the pathophysiological mechanisms in IVD disease in dogs.

\section{References}

1. Bray JP, Burbidge HM. The canine intervertebral disk: part one: structure and function. J Am Anim Hosp Assoc 1998;34: 55-63.

2. Anderson DG, Tannoury C. Molecular pathogenic factors in symptomatic disc degeneration. Spine J 2005;5(Suppl6):S260-6.

3. Gruber HE, Hanley EN, Jr. Analysis of aging and degeneration of the human intervertebral disc. Comparison of surgical specimens with normal controls. Spine 1998;23:751-7.

4. Ghosh P, Taylor TK, Braund KG, Larsen LH. The collagenous and non-collagenous protein of the canine intervertebral disc and their variation with age, spinal level and breed. Gerontology 1976;22:124-34.

5. Melrose J, Taylor TK, Ghosh P. Variation in intervertebral disc serine proteinase inhibitory proteins with ageing in a chondrodystrophoid (beagle) and a non-chondrodystrophoid (greyhound) canine breed. Gerontology 1996;42:322-9.

6. Buckwalter JA. Aging and degeneration of the human intervertebral disc. Spine 1995;20:1307-14.

7. Bray JP, Burbidge HM. The canine intervertebral disk. Part Two: Degenerative changes--nonchondrodystrophoid versus chondrodystrophoid disks. J Am Anim Hosp Assoc 1998;34:135-44.

8. Jensen VF, Christensen KA. Inheritance of disc calcification in the dachshund. J Vet Med A Physiol Pathol Clin Med 2000;47: 331-40.

9. Hunter CJ, Matyas JR, Duncan NA. Cytomorphology of notochordal and chondrocytic cells from the nucleus pulposus: a species comparison. J Anat 2004;205:35762.

10. Erwin WM, Ashman K, O'Donnel P, Inman RD. Nucleus pulposus notochord cells secrete connective tissue growth factor and up-regulate proteoglycan expression by intervertebral disc chondrocytes.
Arthritis Rheum 2006;54:3859-67.

11. Hunter CJ, Matyas JR, Duncan NA. The three-dimensional architecture of the notochordal nucleus pulposus: novel observations on cell structures in the canine intervertebral disc. J Anat 2003;202: 279-91.

12. Ahsan R, Tajima N, Chosa E, et al. Biochemical and morphological changes in herniated human intervertebral disc. J Orthop Sci 2001;6:510-8.

13. Ariga K, Miyamoto S, Nakase T, et al. The relationship between apoptosis of endplate chondrocytes and aging and degeneration of the intervertebral disc. Spine 2001;26:2414-20.

14. Chen B, Fellenberg J, Wang $\mathrm{H}$, et al. Occurrence and regional distribution of apoptosis in scoliotic discs. Spine 2005;30: 519-24.

15. Festjens N, Cornelis S, Lamkanfi M, Vandenabeele P. Caspase-containing complexes in the regulation of cell death and inflammation. Biol Chem 2006;387:100516.

16. Rannou F, Lee TS, Zhou RH, et al. Intervertebral disc degeneration: the role of the mitochondrial pathway in annulus fibrosus cell apoptosis induced by overload. Am J Pathol 2004;164:915-24.

17. Thornberry NA. Caspases: key mediators of apoptosis. Chem Biol 1998;5:R97-R103.

18. Park JB, Lee JK, Park SJ, et al. Mitochondrial involvement in fas-mediated apoptosis of human lumbar disc cells. J Bone Joint Surg Am 2005;87:1338-42.

19. Thornberry NA, Lazebnik Y. Caspases: enemies within. Science 1998;281:1312-6.

20. Duan WR, Garner DS, Williams SD, et al. Comparison of immunohistochemistry for activated caspase- 3 and cleaved cytokeratin 18 with the TUNEL method for quantification of apoptosis in histological sections of PC-3 subcutaneous xenografts. J Pathol 2003;199:221-8.

21. Krayer M, Rytz U, Oevermann A. Apoptosis of ligamentous cells of the cranial cruciate ligament form stable stifle joints of dogs with partial cranial cruciate ligament rupture. Am J Vet Res 2008;69:625-30.

22. Ghosh P, Taylor TK, Braund KG, Larsen LH. A comparative chemical and histochemical study of the chondrodystrophoid and nonchondrodystrophoid canine intervertebral disc. Vet Pathol 1976;13:414-27.

23. McKee M. Intervertebral disc disease in the dog 1. Pathophysiology and diagnosis. In Practice 2000;22:355-69.
24. Zhao CQ, Jiang LS, Dai LY. Programmed cell death in intervertebral disc degeneration. Apoptosis 2006;11:2079-88.

25. Bertram H, Nerlich A, Omlor G, et al. Expression of TRAIL and the death receptors DR4 and DR5 correlates with progression of degeneration in human intervertebral disks. Mod Pathol 2009;22:895-905.

26. Unglaub F, Thomas SB, Kroeber MW, et al. Expression of TRAIL and death receptor DR4 in Palmer type 2 TFCC lesions. Arch Orthop Trauma Surg 2009;130:1215-20.

27. Bursch W, Paffe S, Putz B, et al. Determination of the length of the histological stages of apoptosis in normal liver and in altered hepatic foci of rats. Carcinogenesis 1990;11:847-53.

28. Gruber HE, Ingram JA, Norton HJ, Hanley EN Jr. Senescence in cells of the aging and degenerating intervertebral disc: immunolo-calization of senescence-associated beta-galactosidase in human and sand rat discs. Spine 2007;32:321-7.

29. Ha KY, Koh IJ, Kirpalani PA, et al. The expression of hypoxia inducible factor1alpha and apoptosis in herniated discs. Spine 2006;31:1309-13.

30. Hunter CJ, Matyas JR, Duncan NA. The notochordal cell in the nucleus pulposus: a review in the context of tissue engineering. Tissue Eng 2003;9:667-77.

31. Horwitz T. The human notochord: a study of its development and regression, variations, and pathologic derivative, chordoma. Indianapolis. 1977.

32. Walmsley R. The development and growth of the intervertebral disc. Edinb Med $\mathrm{J}$ 1953;60:341-64.

33. Maldonado BA, Oegema TR, Jr. Initial characterization of the metabolism of intervertebral disc cells encapsulated in microspheres. J Orthop Res 1992;10:677-90.

34. Hansen HJ. A pathologic-anatomical study on disc degeneration in dog, with special reference to the so-called enchondrosis intervertebralis. Acta Orthop Scand Suppl 1952;11:1-117.

35. Erwin WM, Ashman K, O'Donnel P, Inman $\mathrm{RD}$. CTGF/CCN-2 is produced by non-chondrodystrophic canine intervertebral discderived notochordal cells and upregulate nucleus pulposus aggrecan gene expression. J Cell Commun Signal 2008;2:101-25.

36. Lee JH, Chung KC, Kim HJ. Disparity between MR imaging and histochemical grading in human intervertebral disc degeneration. J Korean Neurosurg Soc 2006;39:432-7. 